
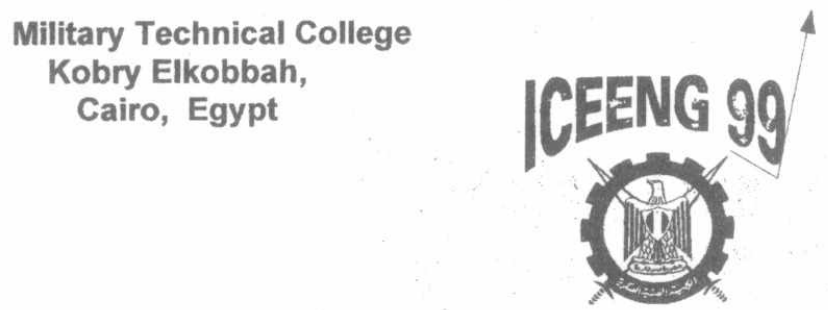
$2^{\text {nd }}$ International Conference on Electrical Engineering ICEENG 99

\title{
AGC DECEPTION: PERFORMANCE ANAL.YSIS AND SELECTION OF JAMMING WAVEFORM PARAMETERS
}

\author{
A. H. ASSEESY ${ }^{1}$
}

\begin{abstract}
This paper analyses the transient and steady state performances of a typical missile receiver AGC system. The analysis is done under the effect of periodic ON-OFF switching of a repeater jamming signal, both in the presence and in the absence of the useful target-reflected signal. Exst formulae are derived for the resulting "blanking times". The most suitable values for the jamming waveform time parameters are deduced for maximum possible suppression of the useful signal mplitude measurement process.
\end{abstract}

\section{KEY WORDS}

Blanking times, IF gain, FB filter, Regulation characteristics, contour s of constant gain, jamming repetition period, jamming duty ratio, suppression ratio.

\section{NOMENCLATURE}

$A G C, 20 \log A_{i}, \Delta S_{J}, \Delta S, \Delta S^{\prime}, S_{v}, V_{s a t}, G_{s a t}, G_{0}, B, e_{r}, t_{b 1}, t_{b 2}, t_{b}$,

\section{INTRODUCTION}

To destroy a target; a homing miss!! . mus! .. ack it:s direction. To track the direction of a certain target it has to be resolved by usirig a selective time-domain or frequencydomain gate. By tracking the basic co-ordinate (range or relative speed); the missile gains additional information that helps the guidance process itself. The main mission of a self protection jammer is to minimize the accuracy of missile guidance by inducing errors in the angular tracking. If the jammer degrades only the basic co-

\footnotetext{
'Ph.D., EW Engineering Department, MTC,
} 
ordinate tracking; the missile may continue its successful operation by tracking the direction of the jammer, which is the same target direction. On the other hand; if the jammer aims only at deceiving the angle measurement without suppressing the target return signal; the existence of the real target return would degrade the effectiveness of angle deception [2]. However, jamming technique parameters can be optimized also in such cases as will be shown in this paper.

In order to measure and track the target direction, the missile has to measure the instantaneous received signal amplitude. In sequential comparison tracking systems the amplitude is compared with its previous values. In instantaneous comparison systems the amplitudes received from two different channels are compared. In either case a correct amplitude measurement is necessary for correct angle measurement. The main goal of this jamming technique is to minimize the periods during which the received signal amplitude can be measured by forcing the AGC of the missile receiver into false control states for the longest possible times which degrades the rate at which the tracking system can follow fast target variations

\section{TYPICAL AGC CHARACTERISTICS}

Fig. 1 shows typical AGC characteristics for a homing missile receiver [1]. In this figure are shown the system functional diagram, the input-output regulated characteristic, the regulated values of the IF gain vs the average output voltage and average input power level $\left(20 \log A_{i}\right)$. Any sudden variation of the system input results in a corresponding output variation without instantaneous gain change; after which the IF gain responds to the new output voltage level; with the time constant of the feedback AGC filter; such that the system returns to a regulated operating point.

\section{TRANSIENT ANALYSIS FOR SLOW JAMMING ON-OFF RATES}

\section{In the Presence of the Reflected Signal}

a. Let the jamming-to-signal ratio be $\Delta_{S}$, at the receiver input. Let the average reflected signal level be $S_{0}[\mathbf{d B m}]$ at the IF amplifier output. Let the IF output level necessary to saturate the video amplifier be $\left(S_{0}+\Delta S\right)$ and that corresponding to the detection threshold level be $\left(S_{0}-\Delta S^{\prime}\right)$. Let the AGC system operating point be initially regulated at the centre of tre A.GC. characteristics (point $C$ in Fig. 1). The initial conditions are:

$v_{o}\left(0_{-}\right)=v_{F}\left(0_{-}\right)=e_{r} \quad, v_{c}\left(0_{-}\right)=0 \quad$ and $G_{I F}\left(0_{-}\right)=G_{0}$

where $v_{0}$ and $v_{F}$ are the video output and FB filter output voltages, respectivey.

b. As the jamming signal arrives; a sudden increase $\Delta S_{J}$ occurs at the input. Since the IF gain can not instantaneously' change; the operating point is transferred; along the constant-gain contour passing through the point "C" (Fig.2) to a temporary point"e". The system is temporarily saturated and the video output equals $\boldsymbol{V}_{\text {sat }}$ if the 
following condition holds:

$$
S_{J}=10 \cdot \log \left(\frac{J+S}{S}\right)>\Delta S
$$

c. The feedback filter output starts to increase towards $V_{\text {sat }}$ with its time constant $\tau_{\text {AGC }}$ as follows:

$$
\left.v_{F}=e_{r}+\left(v_{s a t}-z_{r}\right) \cdot\left(1-e^{-\left(t / \tau_{A G C}\right.}\right)\right)
$$

With the same time constant the control voltage decreases towards $-\left(V_{s a t}-e_{r}\right)$ and the IF gain decreases towards $G_{\text {sat }}=G_{0}-B .\left(V_{\text {sat }}-e_{r}\right)$ :

$$
\begin{aligned}
& v_{c}=-\left(v_{s a t}-e_{r}\right) \cdot\left(1-e^{-\left(t / \tau_{A G C}\right)}\right) \\
& \left.G_{I F}=G_{0}-B \cdot\left(v_{s a t}-e_{r}\right) \cdot\left(1-e^{-\left(t / \tau_{A G C}\right.}\right)\right)
\end{aligned}
$$

Accordingly the IF amplifier output power $[\mathrm{dBm}]$ decreases exponentially with time :

$$
\left.S_{I F}=S_{0}+\Delta S_{J}-B \cdot\left(v_{s a t}-e_{r}\right) \cdot\left(1-e^{-\left(t / \tau_{\text {AGC }}\right.}\right)\right)
$$

d. At a certain time $\boldsymbol{t}_{\boldsymbol{b} 1}$; the IF amplifier arrives at the level where the video amplifier starts to go out of saturation (the temporary point " $f$ "). At this point:

$$
\left.S_{I F}=S_{0}+\Delta S_{J}-B \cdot\left(v_{s a t}-e_{r}\right) \cdot\left(1-e^{-\left(t_{b 1} / \tau_{A G C}\right)}\right)\right)=S_{0}+\Delta S
$$

from which the first blanking time $t_{b 1}$ can be expressed as:

$$
t_{b 1}=-\tau_{A G C} \cdot \ln \left[1-\frac{\left(\Delta S_{J}-\Delta S\right)}{B \cdot\left(v_{s a t}-e_{r}\right)}\right]
$$

It can be easily shown that the gain at that point is $G\left(t_{b 1}\right)=G_{f}=G_{0}-\left(\Delta S_{J}-\Delta S\right)$

e. At $t>t_{b 1}$ the output voltage goes below $v_{s a t}$. The FB filter output continues to increase but towards values $<v_{\text {sat. }}$. The rate $\left(d v_{F} / d t\right)$ decreases with time until it reaches zero very soon. The reasor: is that the logarithm of the output voltage decreases proportionally with the decrease of the IF gain. Therefore $v_{v}$ decreases with time within this interval more rapidly than the decrease of $G_{I F}$ and the path on the AGC characteristics approaches rapidly the regulated operating point " $H$ ". This path $(f \rightarrow H)$ goes along the constant-input curye passing through the two points (a 


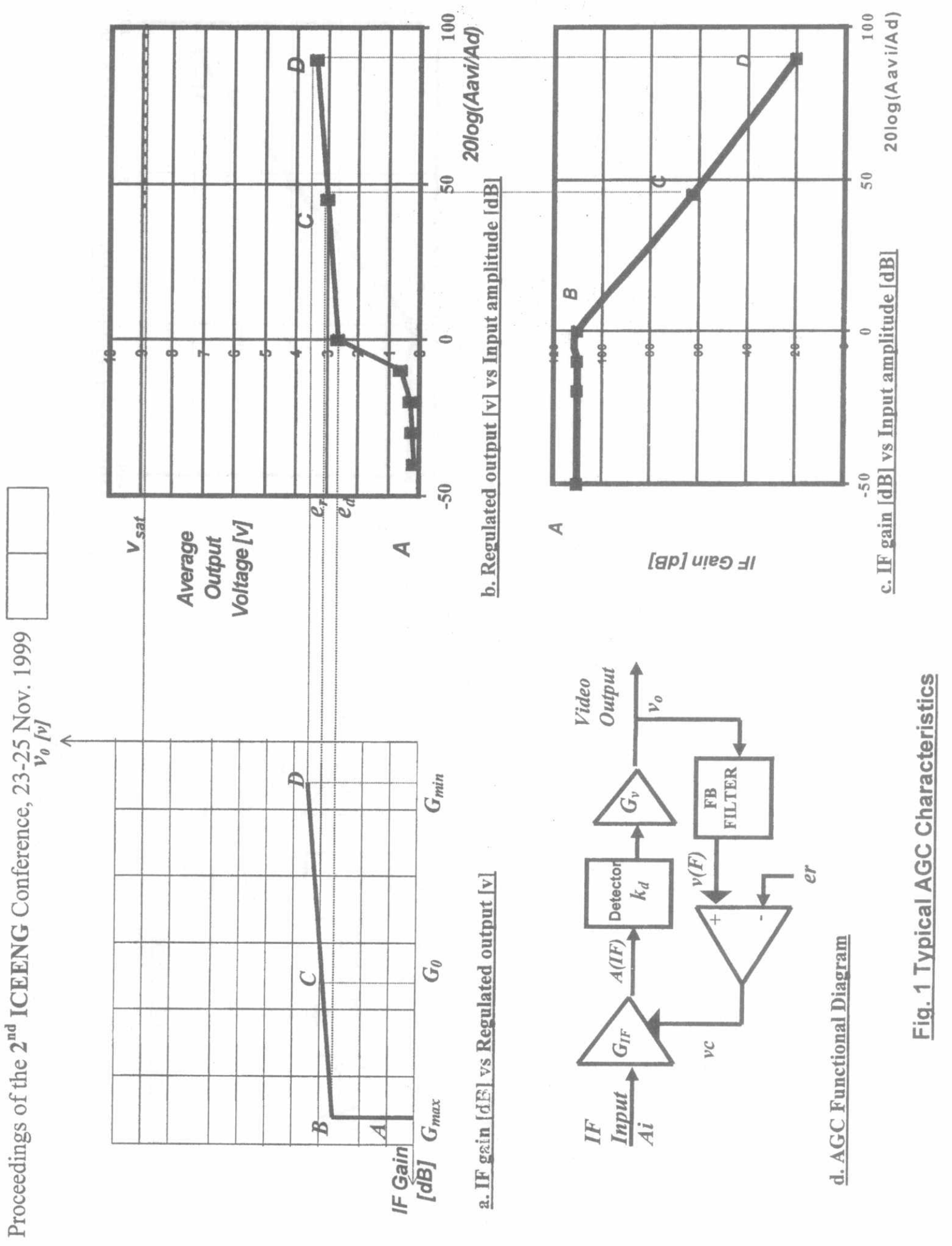




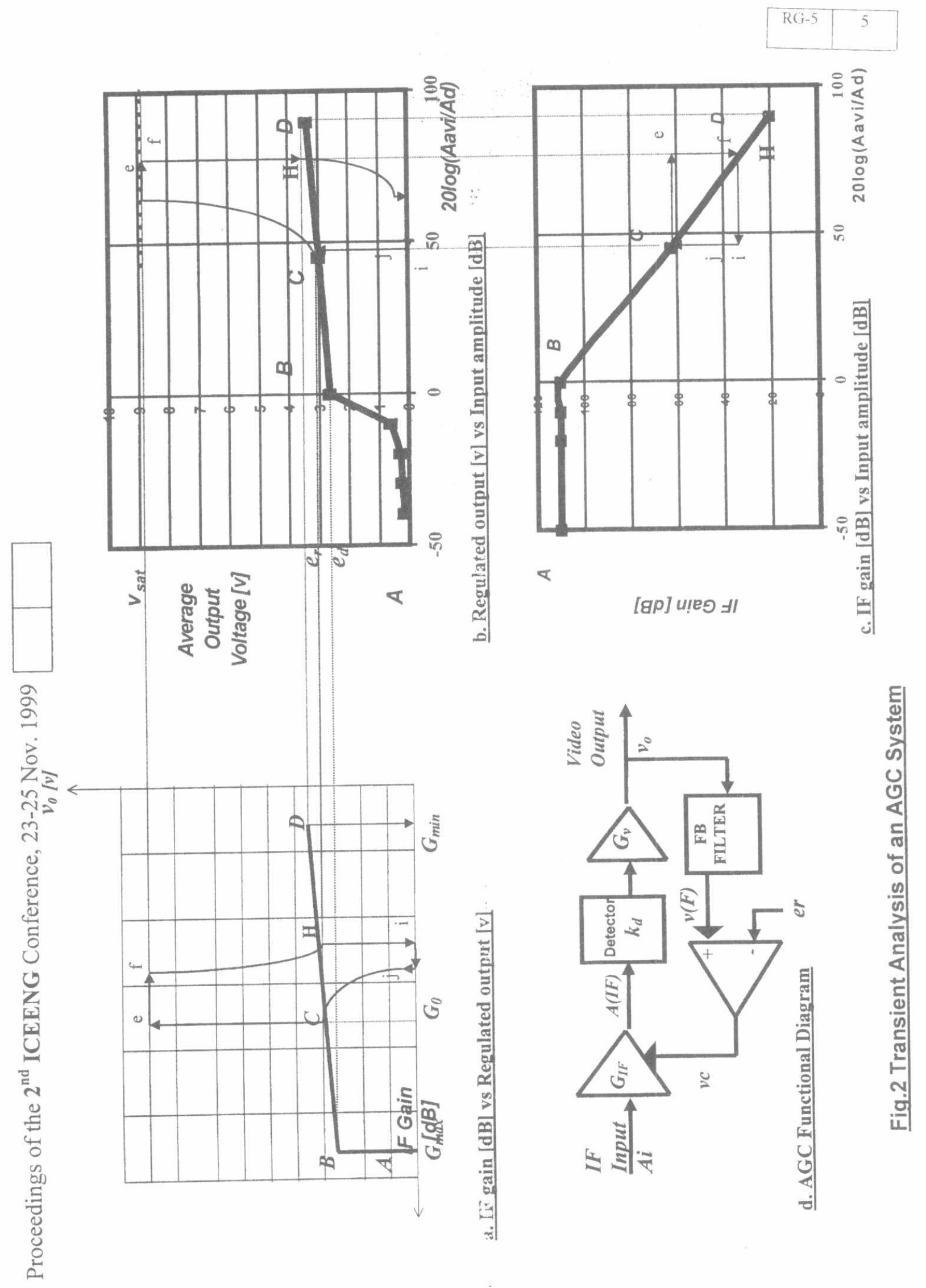


logarithmic curve on the $G_{I F}-V_{V}$ characteristic). The regulated IF gain at this point is $G_{H}=G_{0}-\Delta S_{J}$.

f. At the time instant $t_{2}=\delta_{J} . T_{J}$ the jamming is switched OFF and the input power goes a step $\Delta S_{J}$ down to the reflected signal level. On the constant-gain contour

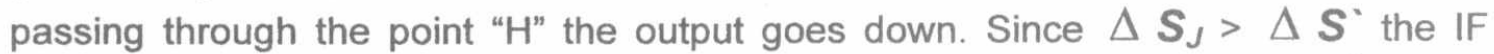
amplifier output amplitude goes below the minimum detectable signal level $A_{\min }$ and the output is cut-off (the temporary point "i").

g. The FB filter starts its slow decrease towards zero and the control voltage increases towards $e_{r}$. The IF gain increases; accordingly, towards the value $\left(G_{0}+\right.$ B. $\left.e_{r}\right)$; at which it will not arrive:

$$
\begin{aligned}
& v_{F}=v_{H} \cdot \exp \left(-\left(t-t_{2}\right) / \tau_{A G C}\right)=\left(e_{r}+\frac{\Delta S_{J}}{B}\right) \cdot \exp \left(-\left(t-t_{2}\right) / \tau_{A G C}\right) \\
& v_{c}=e_{r}-\left(e_{r}+\frac{\Delta S_{J}}{B}\right) \cdot \exp \left(-\left(t-t_{2}\right) / \tau_{A G C}\right) \\
& G_{I F}=G_{0}+B \cdot e_{r}-\left(B \cdot e_{r}+\Delta S_{J}\right) \cdot \exp \left(-\left(t-t_{2}\right) / \tau_{A G C}\right)
\end{aligned}
$$

where $V_{H}$ is the output voltage at the point " $\mathrm{H}$ ". Thus the IF amplifier output power [in $\mathrm{dBm}]$ will increase as follows:

$$
S_{I F}=\left(S_{i}\right)+\left(G_{I F}\right)=S_{0}+B \cdot e_{r}-\left(B \cdot e_{r}+\Delta S_{J}\right) \cdot \exp \left(-\frac{\left(t-t_{2}\right)}{\tau_{A G C}}\right)
$$

h. At a certain time $\left(\boldsymbol{t}_{3}=\Delta \delta \mathrm{J} \cdot \boldsymbol{T}_{\mathrm{J}}+\boldsymbol{t}_{\mathrm{b} 2}\right)$ the IF amplifier output reaches the detection threshold level $\left(S_{d}-\Delta \Delta S^{*}\right)$ and the reflected signal amplitude information is once more available (the temporary point " $j$ "). Equating the IF signal level at the time $\left(t_{2}+t_{b 2}\right)$ with the detection threshold $\left(S_{d^{\prime}}-\Delta S^{\prime}\right)$; we get the second blanking time:

$$
t_{b 2}=-\tau_{A G C} \cdot \ln \left[\frac{\Delta S^{\prime}+B \cdot e_{r}}{\Delta S_{J}+B \cdot e_{r}}\right]
$$

I. At time $>\mathrm{t} 3$ the filter output continues its decrease but towards the new positive value of $v_{v}$ at a decreasing time rate. As the filter output increases the IF gain increases and; consequently, the output increases; which causes the rate of decrease of the filter output to decrease further; until this rate reaches zero at the regulated point " $C$ ". The path $(j \rightarrow C)$ goes along the constant-input curve passing through the two points (a logarithmic curve on the $G_{I F}-V_{V}$ characteristic). 


\section{In the Absence of the Reflected Signal}

a. Due to the success of gate stealing; only the jamming signal exists at the missile receiver input. The initial conditions rorrespond to the point " $\mathrm{H}$ " on the AGC characteristics. At $t=0$ the jamming is switched OFF and no signal is received. The operating point is instantaneously shifted to point " $p$ " along the constant gain contour passing through the stable operating point " $\mathrm{H}$ ". At that point both the input and the output are zero and the gain is $G_{H}=G_{0}-\Delta S_{J}$; where $\Delta S_{J}$ is the opewr difference [in $d B]$ between the jamming signal and the centre of the AGC characteristics.

b. Since the output becomes zero; the F.B. filter output starts its discharge towards zero. At the end of the OFF time period $t_{O F F}=\left(1-\delta_{J}\right) T_{j}$; it arrives at a value $v_{F 1}$ :

$$
v_{F 1}=\left(e_{r}+\frac{\Delta S J}{B}\right) e^{-\left(t_{D F F} / \tau_{A G C}\right)}
$$

The gain arrives at some value $G_{1}$ dependent on this OFF time period:

$$
G_{1}=G_{0}+B \cdot\left(e_{r}-v_{F_{1}}\right)
$$

If the OFF time period is long enough the gain arrives at its maximum value $G_{\max }$ and the operating point arrives at the stable point " $A$ ". The condition is that:

$$
\left(1-\delta_{J}\right) \cdot T_{J} \geq-\tau_{A G C} \cdot \ln \left(\frac{G_{0}+B \cdot e_{r}-G_{\max }}{\Delta S_{J}+B \cdot e_{r}}\right)
$$

c. At the end of the jamming repetition period $T_{J}$ the jamming is switched $O N$ again and the IF output is given by the sum of the input jamming power $\mathrm{J}[\mathrm{dBm}]$ and the IF gain $G_{1}$. The IF amplifier will be saturated if this output exceeds the saturation level; i.e. if:

$$
S_{0}-G_{0}+\Delta S_{J}+G_{1}>S_{0}+\Delta \dot{\omega}
$$

Substituting for $G_{1}$ in (13) we get the condition for saturation:

$$
\left(1-\delta_{\jmath}\right) \cdot T_{\jmath}>-\tau_{A G C} \cdot \ln \left[1-\left(\frac{\Delta S}{\Delta S_{\jmath}+B \cdot e_{r}}\right)\right]
$$

This condition is very likely to be fulfilled; especially if $\Delta S=20 \log \left(V_{\text {sat }} / \mathbf{e}_{r}\right)<<B . e_{r}$ which is usually the case. The operating point instantaneously moves along the constant gain contour passing through the points " $A$ " and "B. It continues to the temporary point " $r$ " at which the receiver is still saturated.

d. The filter output tends towards $\boldsymbol{V}_{\text {sat }}$, the control voltage towards $\mathbf{e}_{r}-\boldsymbol{V}_{\text {sat }}$ and the IF gain towards $G_{0}-B .\left(V_{\text {sat }}-e_{r}\right)$ as follows:

$$
G_{I F}=G_{1}-B \cdot\left(v_{s a t}-v_{F 1}\right)\left[1-e^{-\left(t-t_{\text {OFF }}\right) / \tau_{A G C}}\right]
$$


When the gain arrives at a certain critical value $G_{0}+\Delta S-\Delta S_{J}$; the system goes out of saturation. Equating the gain with its critical value; we get the blanking time:

$$
t_{b}=-\tau_{A G C} \cdot \ln \left[1-\frac{\left(\Delta S_{J}-\Delta S+G_{1}-G_{0}\right)}{B \cdot\left(v_{s a t}-v_{F 1}\right)}\right]
$$

Substituting for $\mathrm{G}_{1}$ and $\mathrm{v}_{\mathrm{F} 1}$ in (16) we get::

$$
t_{b}=-\tau_{A G C} \cdot \ln \left[\frac{B \cdot\left(v_{\text {sat }}-e_{r}\right)-\left(\Delta S_{J}-\Delta S\right)}{B \cdot\left(v_{\text {sat }}-v_{F 1}\right)}\right]=-\tau_{A G C} \cdot \ln \left[\frac{B \cdot\left(v_{\text {sat }}-e_{r}\right)-\left(\Delta S_{J}-\Delta S\right)}{B v_{\text {sat }}-\left(B e_{r}+\Delta S_{J}\right) e^{-\left(t_{\text {orr }} / \tau_{\text {wax }}\right)}}\right]
$$

If the ON time $\delta_{J} \cdot T_{J}$ is longer than the blanking time; the system returns to its original operating point " $\mathrm{H}$ ". Otherwise; it starts its new cycle at a different operating point.

\section{Optimum Time parameters of the Jamming Waveforms:}

a. In the presence of the reflected signal the bets results are obtained iff:

$$
\delta_{J} T_{J} \cong t_{b 1} \quad \text { and } \quad\left(1-\delta_{J}\right) . T_{J} \cong t_{b 2}
$$

b. In the absence of the reflected signal the bets results are obtained iff:

$$
\delta_{J} T_{J} \cong t_{b}
$$

Since the values of the blanking times depend on the missile receiver system parameters; a study of such dependencies is necessary. Such a study has been done by the author; based on the formulae (6), (9) and (16). The most significant system parameters affecting the three blanking times are the saturation margin $\Delta S$ and the cut-off margin $\Delta s$. However; the two margins can not be increased without limits. In the typical model of this study a practical value for $\Delta S$ has been assumed to be $9[\mathrm{~dB}]$ and $\Delta S^{\prime}$ has been given a $4.8[\mathrm{~dB}]$ practical value. These practical values have been estimated through a study of a typical missile receiver design requirements. For these assumptions and $\mathrm{B}=126.4[\mathrm{~dB} / \mathrm{v}]$; blanking times of the order of $0.04 \tau_{\text {AGC }}$ have been obtained.

\section{STEADY STATE ANALYSIS FOR VERY FAST JAMMING CYCLES}

If $\delta \mathrm{J} . \mathrm{T}_{\mathrm{J}} \ll \mathrm{t}_{\mathrm{b} 1}$ and $\left(1-\delta_{\mathrm{J}}\right) \cdot \mathrm{T}_{\mathrm{J}} \ll \mathrm{t}_{\mathrm{b} 2}$; the IF gain will be adjusted at a certain value corresponding to the average input level. Assuming that the $S / N$ ratio is sufficiently high; we can write the following approximate formula for the average input amplitude: 
$\overline{A_{\text {inp }}} \cong A_{J} \cdot \delta_{J}+A_{S} \cdot\left(1-\delta_{J}\right)=A_{S} \cdot\left[1+\delta_{J} \cdot\left(\sqrt{\frac{J}{S}}-1\right)\right]$

The average IF gain will be :

$\overline{G_{I F}} \cong G_{0}-20 \log \left[1+\delta_{J} \cdot\left(\sqrt{\frac{J}{S}}-1\right)\right][\mathrm{dB}]$

The condition for saturation during the $\mathrm{ON}$ time is:

$J+G_{0}-20 \log \left[1+\delta_{J} \cdot\left(\sqrt{\frac{J}{S}}-1\right)\right]>S+G_{0}+\Delta S$

The condition for complete blanking during the OFF period; even in the presence of the reflected signal, is :

$$
S+G_{0}-20 \log \left[1+\delta_{J} \cdot\left(\sqrt{\frac{J}{S}}-1\right)\right]<S+G_{0}-\Delta S
$$

From (20) and (21) we get the the upper and lower limits of $\delta_{j}$; respectively, for complete suppression of the useful amplitude information in the presence of the reflected signal. These two conditions can be summarized in the following inequality:

$$
\frac{10\left(\frac{\Delta S}{20}\right)-1}{\left(10\left(\frac{J-S}{20}\right)\right)-1}<\delta_{J}<\frac{10\left(\frac{J-S-\Delta S}{20}\right)-1}{\left(10\left(\frac{J-S}{20}\right)\right)-1}
$$

These upper and lower limits of $\delta_{\mathrm{J}}$ are plotted in Fig. 3 for different values of $\Delta S$ and $\triangle \mathbf{S}^{*}$.

From these plots we can deduce the following:

a. If $\Delta S_{J}>30[\mathrm{~dB}]$; a complete suppression is possible for $10 \%<\delta_{J}<20 \%$.

b. For $\Delta \boldsymbol{S}_{J}>40$ [dB]; $\delta \delta J_{\min } \Rightarrow 0$ and $\delta \delta J_{\max } \Rightarrow$ a constant value dependent on $\Delta \mathrm{S}$; which means that the behaviour of the AGC system under jamming approaches that in the case of successful gate stealing. Increasing the saturation margin $\Delta S$ decreases the upper limit of $\Delta \mathbf{S}$ necessary for complete suppression. However, such an increase represents a considerable burden on the missile receiver design.

\section{CONCLUSION}

1. AGC deception is an effective jamming technique against angle tracking systems based on amplitude measurement. It can deny the tracking system the useful amplitude information during certain time periods (called blanking times) along every jamming cycle. Maximization of the ratio between these blanking times and the 
Proceedings of the $\mathbf{2}^{\text {nd }}$ ICEENG Conference, 23-25 Nov. 1999

\begin{tabular}{|l|l|}
\hline $\mathrm{RG}-5$ & 10 \\
\hline
\end{tabular}

jamming repetition period (defined as the suppression ratio $Q_{s}=\Sigma t_{b} / T_{J}$ is the objective of an effective deception technique design.

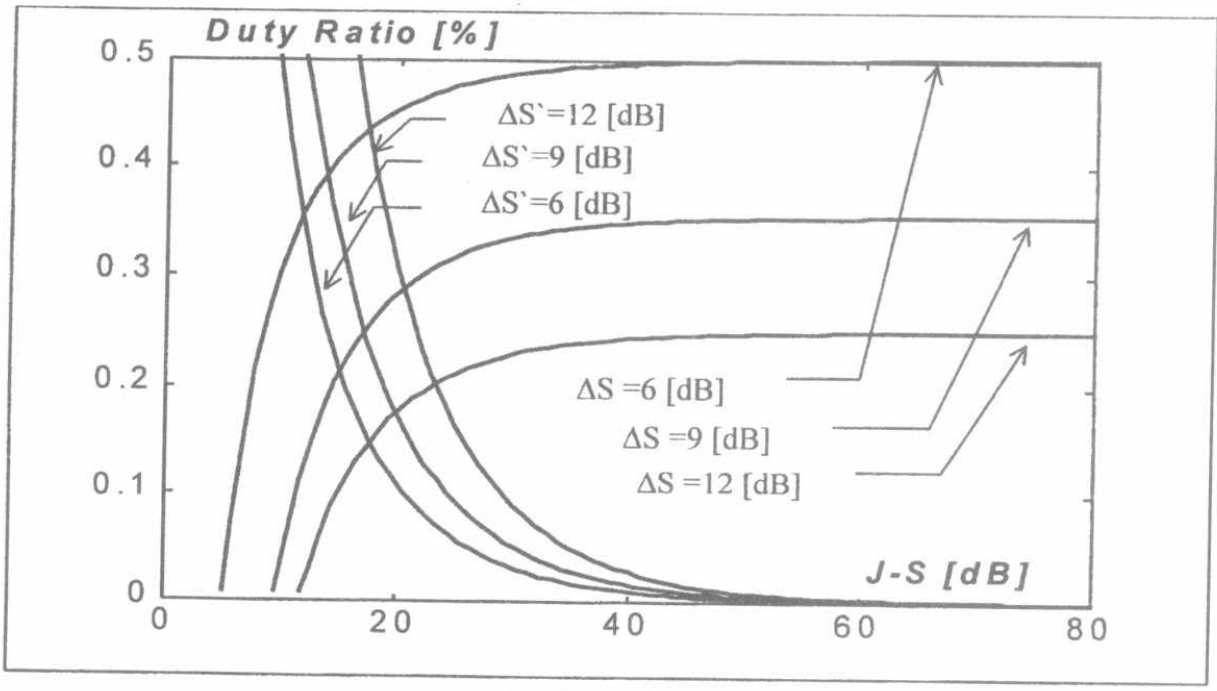

Fig. 3. Upper and lower limits of $\delta$ for complete suppression

2. Since the blanking times are functions of the missile receiver system parameters; the optimization process has to select the best values for the jamming repetition period $T_{J}$ and duty ratio $\delta_{J}$ for maximum possible suppression ratio; assuming different possible values for the AGC system parameters.

3. Although analytical formulae have been derived by the author for the blanking times and the conditions for selecting the jamming duty ratio and in some cases the repetition period; such an optimization process needs a detailed simulation model; in which different system parameters can be varied and their effects on the optimality conditions can be thoroughly studied.

\section{REFERENCES}

1. A. H. Assisi, "Modeling the AGC of a Homing Missile Receiver as an Aid for ECM Evaluation", Proceedings of the $8^{\text {th }}$ ASAT Conference, 1999.

2. L. B. Van Brunt, "APPLIED ECM", Vol.1 \& 3 EW Engineering, (1982-1995). 
